\title{
FENOMENOLOGÍA Y ESENCIA PROCESUAL HUMANA
}

\author{
Pilar Fernández Beites \\ Universidade Pontificia de Salamanca
}

En unas Jornadas como éstas que unen a fenomenólogos de países vecinos, creo que hemos de intentar aunar esfuerzos para abrir caminos futuros en la fenomenología y, por tanto, en la filosofía. Con el fin de contribuir a este intento dedicaré mi ponencia a señalar el ámbito de problemas en el que vengo trabajando desde hace varios años.'

Mi investigación se centra en construir una teoría de la persona. Me parece que hoy más que nunca es necesario hablar de la persona en serio, y esto significa hacer ontología de la persona. En una ponencia anterior, Agustín Serrano de Haro se ocupó de la ontología de la fenomenología y ahora hemos de ver cómo podemos aplicar dicha ontología al ser humano, porque en el hombre encontramos un problema decisivo: el problema del hacerse, del cambio. Para entender a la persona hemos de tener en cuenta su carácter procesual, lo que podemos denominar el "dinamismo" de la vida humana. ${ }^{2}$

Es un dato innegable que las personas van cambiando de modo constante a lo largo de su proceso vital. Yo, desde luego, no soy ahora como era hace diez o quince años. No sólo se ha modificado externamente mi cuerpo (tal como se constata de un golpe de vista), sino que mis hábitos, mis capacidades, mis conocimientos... han sufrido múltiples alteraciones; incluso puedo haber experimentado un cambio radical de carácter, que hace que mis amigos vean ahora en mí una persona nueva. Estos cambios que encontramos en cualquier biografia personal son los que plantean el problema del dinamismo de la vida humana.

\footnotetext{
1 Resumiré aquí las conclusiones de varios ensayos aún no publicados en los que intento justificar con detalle las tesis que defenderé a continuación.

2 La expresión "dinamismo" la tomo de Zubiri que considera decisivo hablar del dinamismo de lo real (cf. Estructura dinámica de la realidad, Alianza Editorial, Madrid, 1989).
} 
Hemos de reconocer que el hombre no es una realidad ya hecha de antemano, sino que ha de hacerse. El agua es agua desde siempre, desde siempre cuenta con su esencia. Pero el hombre ha de intervenir en la construcción de su propia esencia. El individuo humano no nace con una esencia totalmente invariable, sino que en buena medida la vida del hombre consiste en la construcción de su esencia.

Pues bien, en mi opinión, para dar cuenta del dinamismo vital no nos sirve hasta el final la ontología clásica. La razón es que en ella sólo se considera esencial la "sustancia" humana invariable y se relega el cambio al ámbito de lo "accidental". Pero parece que en el caso del hombre es imposible negar el carácter esencial a aquello que cambia. Lo que le sucede al agua o a una piedra no les resulta esencial, pero al hombre no sólo le suceden cosas accidentales, como a la piedra o al agua, sino que muchos de los cambios que el hombre experimenta en su vida son modificaciones que él mismo produce libremente. Y estas múltiples variaciones que vive el hombre en tanto que ser libre tienen que considerarse esenciales, pues en ellas se juega entre otras cosas la dignidad ética de la persona. Tomar en serio la libertad exige afirmar que lo que el hombre hace de sí mismo ha de ser esencial, es decir, los resultados de la libertad han de añadirse a la esencia previa del hombre, de modo que sean también esenciales. Pero esto resulta inconcebible en el esquema clásico en el que todas las modificaciones libres han de ser accidentales: la esencia permanece invariable $-y$, por supuesto, específica, no individual $-{ }^{3}{ }^{3}$

¿Podrá la fenomenología ofrecer un esquema ontológico alternativo al clásico en el que la esencia incorpore el hacerse? Mi opinión es que sí.

\section{Existencialismo y esencia humana}

El dato básico acerca del carácter procesual de la vida humana ha sido sacado a la luz por el existencialismo de Martin Heidegger. Heidegger resalta la importancia de la existencia, del ir haciéndose a sí mismo. Pero, como es bien sabido, Heidegger va más lejos y sostiene que la esencia del hombre consiste en la mera exiștencia. El hombre es, para Heidegger, ser-ahí (Da-sein), existencia. No es sólo que la existencia sea crucial para entender al hombre, sino que la existencia sustituye a la esencia: "La 'esencia' del ser ahí reside en su existencia (Existenz)". ${ }^{4}$ Los entes no humanos tienen ser, esencia, pero al

3 El tema de la individuación y, en concreto, de la esencia individual en Xavier Zubiri, lo he tratado en "Sobre la esencia individual", Diánoia, 54, pp. 33-54, México, 2005.

4 Sein und Zeit, Max Niemeyer Verlag, Tübingen, 1986, \&9, p. 42. Trad. cast. El ser y el tiempo, FCE, Madrid, ${ }^{2} 1971$. A partir de ahora me referiré a esta obra con la abreviatura SZ. He de advertir que en este trabajo he modificado todas las traducciones siempre que lo he considerado conveniente.

El texto citado continúa así: "Los caracteres que pueden ponerse de manifiesto en este ente no son, por ende, 'peculiaridades' ante los ojos de un ente ante los ojos de tal o cual 
hombre le corresponde, no el tener ser, sino el tener que ser, no la esencia, sino la existencia. El hombre no tiene "propiedades (Eigenschaften)" como las cosas, sino "posibles modos de ser (mögliche Weise zu sein)." El "qué (Was-sein)" del hombre (esencia) no consiste en un "qué" de contenido material, como mesa, silla, sino que consiste en el "ser relativamente a (Zu-sein)" sus posibilidades, en el tener que hacerse eligiendo entre sus posibilidades (existencia) $^{.5}$

De esta forma, Heidegger elimina la esencia del hombre a favor de una existencia que fácilmente se convierte en un mero acontecer del ser; un acontecer no subjetivo, no humano. Pues bien, frente a esta tesis heideggeriana, creo que hay que afirmar que el hombre posee una esencia - y, además, una esencia estrictamente individual - que no se agota en la mera existencia. El problema es que la ontología que nos permita hablar de una esencia que incorpore la existencia está aún por hacer. En mi opinión, el reto fundamental al que se enfrenta la filosofia en el siglo XXI es construir una teoría de la persona que asuma la tesis heideggeriana, pero superando al mismo tiempo el existencialismo. Ésta es la cuestión. Se trata de no renunciar a la esencia, pues el siglo recién finalizado ya nos ha ofrecido una muestra más que suficiente de teorías que eliminan la esencia y con ella a la persona: postmodernismos, estructuralismos, desconstruccionismos... y existencialismos.

Para mostrar la gravedad del problema que tenemos entre manos empiezo por referirme a un gran fenomenólogo como es Max Scheler, pues creo que Scheler tiene el mérito de haber criticado avant la lettre la tesis existencialista, mediante su radical oposición a la "libertad de indiferencia".

Scheler ha mostrado que una libertad auténtica, que no sea de indiferencia, ha de estar abierta al ámbito de los valores. El hombre no decide de modo azaroso, arbitrario, sino orientado por valores: valores estéticos, religiosos, vitales o incluso los valores de lo meramente agradable-sensible... Como desde la fenomenología nos interesan los valores, pero en su modo de donación, hemos de preguntar por la estructura subjetiva que proporciona la apertura a dicho ámbito de valores. Pues bien, esta apertura es lo que Scheler denomina "ordo amoris" normativo (individual). El ordo amoris normativo es

'aspecto', sino modos de ser posibles para él en cada caso y sólo esto. Todo ser así de este ente es primariamente ser. De donde que el término ser ahí, con que designamos este ente, no exprese su 'qué', como mesa, casa, árbol, sino el ser" ibid.

5 "El ser mismo relativamente al cual puede conducirse y se conduce simpre de alguna manera el ser ahí, lo llamamos existencia (Existenz). Y porque la definición de la esencia de este ente no puede darse indicando un qué de contenido material, sino que su esencia reside en que tiene que ser en cada caso su ser como ser suyo (es je sein Sein al seiniges zu sein hat), se ha elegido para designar este ente el término ser ahí, que es un término que expresa puramente el ser.

El ser ahí se comprende siempre a sí mismo partiendo de su existencia, de una posibilidad de ser él mismo o no él mismo", $S Z, \& 4$, p. 12.

"La 'esencia' de este ente está en su ser relativamente a (in seinem Zu-Sein)", SZ, §9, p. 42 
una apertura afectiva al ámbito de los valores, y una apertura individual, de forma que cada hombre tiene su peculiar ordo amoris, su singular modo de acceder al reino de los valores: el artista verá sobre todo valores estéticos, el filósofo quedará sobrecogido por el valor de la verdad... Y este peculiar ordo amoris que tiene cada persona constituye justamente la esencia humana individual que marca a cada hombre una vocación, un destino individual e intransferible que da cuerpo a la libertad. ${ }^{6}$

La libertad sin esencia, sin ordo amoris es "libertad de indiferencia", es un simple acontecer del ser, un puro azar, que, por tanto, no es libertad, sino una mera nada. Por ello creo que la filosofia de Heidegger - y casi toda la filosofia actual que se nutre, aún sin saberlo, del manantial heideggeriano - es, en el fondo, un nihilismo (que el primer Heidegger encubre parcialmente mediante una bien construida ambigüedad). ${ }^{7}$ Pero defender una libertad que no sea finalmente nihilista nos plantea un problema ontológico de primera magnitud, pues necesitamos una nueva ontología capaz de hablar de la libertad, del dinamismo del hacerse, sin por ello perder la esencia humana.

\section{Ontología de la persona}

Lo que me gustaría mostrar aquí es que la fenomenología tiene la fuerza suficiente para enfrentarse con el difícil problema ontológico que tenemos entre manos. Para ello hemos de volver a las raíces de la fenomenología, pues creo que lo que debemos utilizar es la teoría de todos y partes que desarrolló el fundador de la fenomenología en la tercera investigación lógica. En mi opinión, esto es lo que hace precisamente un filósofo español que ha de ser contado en la lista de los fenomenólogos: Xavier Zubiri. Mi hipótesis interpretativa es que la nueva ontología elaborada por Zubiri en Sobre la esencia es justamente una teoría de todos y partes del tipo de la propuesta por Edmund Husserl. ${ }^{8}$ Merece la pena que nos detengamos a estudiar las tesis de Zubiri, pues se trata de uno de los pensadores que mejor ha detectado el exceso heideggeriano de eliminar la esencia para transformarla en existencia. Además, ha intentado construir una alternativa suficiente al existencialismo, pero de

6 Sobre el ordo amoris normativo y su diferencia con el descriptivo trato en "Ordo amoris y esencia de la persona" (próxima publicación).

7 Hannah Arendt no duda en hablar del nihilismo heideggeriano (CF. “¿Qué es la filosofia de la existencia?", en Hannah Arendt, Ensayos de comprensión. 1930-1954, Caparrós, Madrid, 2005, pp. 203-231). Por mi parte, intentaré justificar esta tesis en un próximo trabajo que muestre como en la ontología heideggeriana el "estado de resuelto" no es realmente una opción existencial, pues el enfrentamiento con la nada del mundo y con nuestra propia nada sólo puede vivirse momentáneamente en la angustia y ha de lanzarnos necesariamente de nuevo a la existencia impropia de la cotidianidad.

8 Justifico con detalle esta tesis en: "Teoría de todos y partes: Husserl y Zubiri", Signos filosóficos, en prensa. 
modo sorprendente su propuesta apenas ha sido tomada en consideración por autores posteriores.

Zubiri habla del dinamismo de la vida humana, mas sin perder la esencia. Afirma, frente a todos los existencialismos, que el hombre sólo puede hacer su propia vida libremente, sólo puede ser existencia, porque no es sólo existencia, sino también esencia. El hombre posee una esencia en sentido fuerte, que no puede agotarse en el hacerse como tal: el hacerse sólo es posible si hay algo que permita el hacerse ( $\mathrm{y}$ es obvio que este algo no puede ser a su vez un hacerse). El hombre no es un mero proceso, un acontecer, sino una "esencia" que se hace a sí misma. Una esencia "abierta", que se distingue de los demás entes que son esencias "cerradas". El hombre está abierto a la realidad, en concreto, a su propia realidad y por ello se comporta operativamente respecto a ella, es decir, actúa libremente. ${ }^{9}$

Lo que demuestra Zubiri es que el que la esencia del hombre dé cabida a la libertad no impide, sino más bien exige que la esencia sea previa a la existencia. La importancia del hacerse obliga a afirmar la esencia, porque es la esencia precisamente la que incluye el tener que hacerse. Como sostiene $\mathrm{Zu}-$ biri con toda claridad: "No hay prioridad de la existencia sobre la esencia, sino que se trata de una esencia que 'de suyo' se comporta operativamente respecto a su propia realidad, porque, y sólo porque, es una esencia transcendentalmente abierta. Una cosa es estar abierto a su propia realidad; otra muy distinta que la esencia se determine procesualmente desde el mero acto de existir. Esto último es metafísicamente imposible". ${ }^{10}$

En definitiva, Zubiri entiende al hombre como un hacerse continuo en el que, sin embargo, no se pierde la esencia, la identidad. Para expresar esta idea, Zubiri logra dar con una fórmula brillante: el hombre es siempre "el mismo", pero no es siempre "lo mismo". El hombre es proceso y, por tanto, es distinto en cada fase del decurso vital: no es siempre lo mismo. Así recogemos el carácter dinámico de la vida. Mas, a pesar de los cambios propios de cada etapa genética, afirmamos que el hombre es siempre el mismo y con ello ponemos el acento en la identidad.

La fórmula de ser el mismo sin ser lo mismo la aplica Zubiri a los seres vivos en general. Frente a la mera "estabilidad" de los entes inanimados, los seres vivos se caracterizan por la "mismidad". Las cosas son estables, son siempre lo mismo. Un ser vivo, sin embargo, no es siempre lo mismo, no posee identidad como estabilidad, pero es siempre el mismo: posee identidad como mismidad. Zubiri muestra, además, en un análisis brillante, que las cosas mantienen su identidad (entendida como estabilidad) a pesar del cambio,

9 Su apertura, en primer lugar intelectiva, le hace abierto también en el sentido que aquí más nos interesa, que es el de abrirse a sus posibilidades futuras (abierto porque no está hecho, sino que ha de hacerse).

10 Sobre la esencia, Alianza Editorial, Madrid, 1985, p. 506. A partir de ahora me referiré a esta obra con la abreviatura $S E$. 
mientras que los seres vivos conservan la identidad (definida ya como mismidad) justamente gracias al cambio. Un ente material es siempre lo mismo a pesar de las acciones del medio sobre él: la piedra es la misma a pesar del fuerte golpe que acaba de sufrir. Por el contrario, el ser vivo es el mismo, no a pesar de, sino gracias a sus acciones en el medio, por ejemplo, gracias a sus constantes funciones metabólicas ${ }^{11}$.

En la persona, el dato de ser el mismo sin ser lo mismo queda recogido mediante la distinción terminológica entre la "personalidad" y la "personeidad". A lo largo de la vida varía la personalidad del individuo, pero esta variación sólo es posible porque hay algo que ha permanecido. Para referirse a esto que permanece, Zubiri introduce un neologismo y habla de "personeidad". Aunque la personalidad cambie, la personeidad no se modifica.

Pasemos ya al ámbito ontológico que nos permite hacer todas estas afirmaciones. En él creo que hemos de hablar de "esencias procesuales o dinámi-

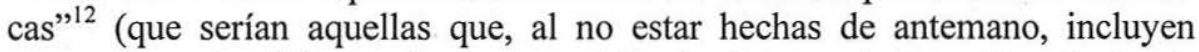
necesariamente el hacerse) y para explicarlas hemos de recurrir a la teoría propuesta por Zubiri en Sobre la esencia. En esta obra Zubiri desarrolla una teoría de todos y partes, pues defiende una "teoría de la sustantividad" frente a la clásica "teoría de la sustancia" y una sustantividad es justamente un todo.

En efecto, Zubiri sostiene que una sustantividad es un sistema clausurado y total de notas constitucionales ${ }^{13}$ (cuya razón formal es la suficiencia constitucional). El sistema se califica de "total" justamente porque las notas que lo estructuran están reguladas por una unidad interna (previa) que es la propia de los todos, frente a los meros agregados. Y la independencia del todo, su carácter de todo final, queda recogido en la noción de clausura. Lo peculiar de la sustantividad frente a la sustancia clásica consiste entonces precisamente en ser un todo que posee partes - las "notas" del sistema -. Por tanto, para Zubiri la estructura básica de la realidad no es la de sustancias que soportan accidentes, sino la de sustantividades compuestas por partes. Así el esquema sustancia-accidente deja paso al de sustantividad-elemento insustantivo (todo-parte).

Pero si queremos pensar a fondo sobre la noción de parte, que es esencial en la teoría de la sustantividad, hemos de acudir, sin duda, a Husserl. Como es sabido, en la tercera de sus investigaciones lógicas, desarrolla Husserl una ontología formal en la que muestra la necesidad de distinguir dos tipos de partes: las no independientes (también denominadas partes abstractas o mo-

11 Cf. Estructura dinámica de la realidad, p. 185 y p. 200.

${ }_{12}$ Zubiri se refiere al hombre como "esencia abierta" y, por mi parte, introduzco el término más amplio de "esencia procesual o dinámica", aplicable también a los seres vivos.

13 En Sobre el hombre, Alianza Editorial, Madrid, 1986, p. 46, Zubiri define la sustantividad como sistema de notas constitutivas, en lugar de constitucionales. 
mentos) y las independientes (llamadas también partes concretas o pedazos) ${ }^{14}$. Las partes no independientes son las que no pueden existir fuera del todo y las independientes las que sí pueden existir fuera del todo. Las partes en el sentido habitual del término son las independientes, pues son las que despedazan el todo, las que lo rompen en pedazos. No puedo quebrar un campo visual en color y extensión - éstas son partes dependientes -, pero sí puedo desmembrar el cuerpo de un animal y obtener su corazón, su cabeza.... - éstas son partes independientes -.

Pues bien, la definición de sustantividad propuesta por Zubiri corresponde a la de un todo formado principalmente por partes independientes, por las partes en sentido habitual. Aunque habría que sostener que la sustantividad tiene también partes dependientes. En realidad el no distinguir explícitamente ambos tipos de partes crea dificultades profundas en algunos puntos de la teoría zubiriana. Pero en esto no puedo entrar aquí.

Veamos ya cómo la teoría de todos y partes hace posible entender el dinamismo humano. En el esquema clásico, la esencia se asocia con la "sustancia" y lo variable es "accidental". Por el contrario, en el nuevo esquema, la "sustancia" invariable se sustituye por la "sustantividad", que es un todo formado por partes invariables y variables, de modo que la articulación de dichas partes va a permitir explicar el dinamismo.

En efecto, Zubiri distingue tres tipos de partes o notas en la sustantividad. En primer lugar, tenemos las "notas adventicias", que corresponden a los clásicos "accidentes". En segundo lugar, están las "notas constitutivas", que serían las notas esenciales en el sentido estricto de la tradición. Pero Zubiri introduce un tercer tipo de notas que creo que son las realmente interesantes: se trata de las "notas constitucionales". Si no me equivoco, el mejor instrumental teórico del que disponemos en la actualidad para explicar el dinamismo de la esencia humana es justamente la distinción "constitutivo-constitucional" propuesta por Zubiri. Pero esta distinción debe ser pensada más a fondo de lo que se atreve a hacerlo el mismo Zubiri.

Mi propuesta es tomar en serio la afirmación de que lo constitucional no es accidental (adventicio), porque creo que esto sólo puede significar, aunque Zubiri no llegue a sostenerlo explícitamente, que lo constitucional es esencial. ${ }^{15} \mathrm{La}$ esencialidad de lo constitucional tiene una importancia decisiva, porque es justamente lo que nos permite dar cuenta de las esencias procesuales. Efectivamente, las notas constitucionales son notas que varían - al contrario que las constitutivas - y de este modo incorporan los cambios de la libertad en la propia esencia. Las notas constitucionales son esenciales, si bien en un

14 Sobre la teoría husserliana de todos y partes, el mejor estudio en castellano es el de Agustín Serrano de Haro, Fenomenología trascendental y ontología, Ed. Universidad Complutense de Madrid, Madrid, 1990.

$15 \mathrm{Y}$ es esencial porque así lo determina la esencia constitutiva. 
nuevo sentido distinto de la tradición, pues se trata de un nivel esencial (no meramente accidental), pero variable. ${ }^{16}$

Con este utillaje ontológico podemos explicar la distinción antes señalada entre la personeidad y la personalidad. La personeidad es lo que soy de siempre, es la esencia en el sentido clásico, la esencia dada de antemano, lo constitutivo. Y la personalidad es lo que voy haciendo de mí a lo largo de mi proceso vital. Esto que voy haciendo de mí no es meramente accidental, sino que debemos entenderlo como esencial en un nuevo sentido: es la esencia que se hace, lo constitucional. ${ }^{17}$

Aquí no puedo justificar hasta el final estas afirmaciones, pues necesitaría mucho más tiempo del que dispongo. ${ }^{18}$ Pero no quiero terminar mi exposición sin indicar en qué sentido la ontología zubiriana permite resolver el problema que ha dejado sobre el tapete el existencialismo de Heidegger.

\section{M. Heidegger y el ser relativamente a la muerte}

También el primer Heidegger parece creer que para explicar el hacerse propio del hombre lo más acertado es recurrir a una teoría de todos y partes del tipo de la propuesta por Husserl. Esto se hace patente cuando en Ser y tiempo introduce en primer plano el tema de la muerte. Aunque sus múltiples intérpretes no suelan indicarlo, el estudio heideggeriano de la muerte se puede entender como el esbozo de una teoría de todos y partes capaz de dar cuenta del todo humano.

En efecto, es al plantear el problema de la totalidad del ser ahí cuando surge el fenómeno de la muerte, que muestra que el ser ahí es un todo muy peculiar. En este punto Heidegger remite ${ }^{19}$ explícitamente en una nota a la

${ }^{16}$ En Josef Seifert encontramos quizás el intento actual más serio por mantener la sustancia clásica (cf. Das Leib-Seele Problem und die gegenwärtige philosophische Diskussion, Wissenschaftliche Buchgesellschaft, Darmstadt, 2., korrigierte und erweiterte Auflage, 1989 y Sein und Wesen, Universitätsverlag C. Winter, Heidelberg, 1996). Pero J. Seifert también propone incluir el cambio en la sustancia (Das Leib-Seele Problem und die gegenwärtige philosophische Diskussion. pp. 115-6, Sein und Wesen p. 159ss) y sostiene que no toda relación de inherencia es accidental (Das Leib-Seele Problem und die gegenwärtige philosophische Diskussion, p. 114): las notas inherentes no accidentales son las que aquí denominamos constitucionales. Sin embargo, creo que lo decisivo es que aceptar esto supone romper el esquema clásico de sustancia-accidente y exige, por tanto, elaborar una nueva teoría ontológica.

17 Así vamos más allá de Zubiri, pues en las cuatro últimas páginas de Sobre la esencia, Zubiri considera lo adquirido por libertad como adventicio (no como constitucional).

${ }^{18}$ En otro trabajo mostraré con detalle qué tipo de fundamentación y de necesidad liga las notas constitucionales con las constitutivas. Zubiri habla de "necesidad lógica" y "necesidad natural"; pero, además, habrá de introducirse explícitamente el tema de la libertad que exige un tipo distinto de fundamentación. 
teoría de Husserl sobre todos y partes de la tercera investigación lógica. Y podemos decir, por tanto, que lo que intentaría mostrar Heidegger es que esta teoría husserliana al ser aplicada al todo humano cobra un sesgo sorprendente. El hombre es un todo muy peculiar, pues al ser un todo que se hace, en él hemos de incluir lo que Heidegger denomina el todavía-no (Noch-nicht), hemos de incluir las partes futuras, las múltiples posibilidades y, por tanto, la muerte como la posibilidad última (la "posibilidad más propia"). Veamos cómo describe Heidegger el todo humano abierto a la muerte, porque se trata de una pieza maestra de la descripción fenomenológica.

En los entes distintos del hombre podemos utilizar una teoría de todos y partes que responde al esquema clásico más habitual. La $\operatorname{cosa}^{20}$ está formada por tales y cuales partes. Si posee todas sus partes está completa; mas puede carecer de alguna de ellas y entonces decimos que esa parte le falta. Esta noción de "falta (Ausstand ( $^{21}$ )" es decisiva para entender los todos clásicos incompletos. El ejemplo elegido por Heidegger es el de una deuda. Supongamos que al ir a sacar dinero del cajero automático compruebo que estoy en números rojos: debo 100 euros. Entonces los 100 euros faltan en mi cuenta. Pero los puedo ir ingresando paulatinamente. Cuando ingreso 30 euros faltan sólo 70 y si logro ingresar los últimos 70 euros ya no falta nada; la deuda está saldada, el descubierto de la cuenta ha podido finalmente cubrirse. En este caso, lo que no-es (o mejor, el todavía-no) tiene el sentido negativo de "faltar": a la cuenta le faltan 100 euros, como a un perro le puede faltar una pata o a un libro una de sus hojas.

Por el contrario, lo peculiar del ser ahí, del hombre, es que el todavía-no no es lo que le falta al todo para estar completo, sino que el todavía-no pertenece al todo, le pertenece en tanto que todavía-no. El todo no tiene sólo las partes actuales, sino también las posibles. Esto es lo peculiar del hacerse, de la existencia. El todo es ya su no-ser, su todavía-no. En el hombre, el no-ser no tiene un sentido negativo, no es lo que falta, sino que está incorporado al propio hombre. Como dice Heidegger de modo magistral, el ser ahí es en cada caso ya su todavía-no. ${ }^{22}$

Esta fuerte tesis que nos obliga a modificar la noción clásica de todo es justificada por Heidegger de modo brillante a través del análisis de la muerte. El ser ahi no es un todo en sentido clásico, al que le falten sus partes futuras, precisamente porque la incorporación de todas sus partes no es otra cosa que la muerte. La totalidad del hombre no se puede lograr entonces nunca con un esquema clásico. Y lo interesante es que lo que falla en este punto no es el hombre, no sucede que el hombre sea un ente defectuoso incapaz de alcanzar

20 Como es sabido, Heidegger no habla en este punto de cosas - "ser ante los ojos (Vorhandenheit)" -, sino de "ser a mano (Zuhandenheit)".

21 SZ., $\$ 48$.

22 "Análogamente, es también el ser ahí, mientras es, en cada caso ya su 'todavía no" (SZ., $\S 48$, p. 244). "El ser ahí es en cada caso su posibilidad" (SZ., §9, p. 42). 
su totalidad, sino que lo que falla es el mismísimo esquema clásico de todos y partes. Hay que modificar entonces dicho esquema en el sentido ya indicado: en el hombre el no-ser está incorporado en tanto que no-ser, la muerte está incorporada como posibilidad. Ésta es la tesis del "ser relativamente a la muerte (Sein zum Tode)". Ella nos permite afirmar que en cualquier momento el hombre está completo, es un todo, porque sus partes futuras le pertenecen en tanto que todavía-no. La muerte pertenece al hombre, no cuando se produce (pues entonces ya no hay ser ahí), sino en tanto que se puede producir: "'Morir (Sterben)' será el término para el modo de ser en que el ser ahí es relativamente a su muerte ( $z$ u seinem Tode ist)". ${ }^{23}$

Así se soluciona la paradoja de Epicuro. Es sabido que para tranquilizar acerca de la muerte este autor decía que mientras nosotros somos, la muerte no es y cuando la muerte es, nosotros no somos. Se trata de un razonamiento en apariencia correcto, pero lo cierto es que no logra tranquilizarnos. Y la razón reside en que el hombre es un ser relativamente a la muerte. En efecto, en la fórmula de Epicuro la muerte es la muerte como acontecimiento intramundano. Es la muerte que pertenece a lo que Heidegger denomina el ámbito de la "impropiedad (Uneigentlichkeit)"; en realidad es la muerte de los otros: cuando Juan es, la muerte no es y cuando la muerte es, Juan ya no es. O dicho de modo más preciso, es la muerte del "se (man)". "Se" muere alguien, incluso todos "se" tienen que morir..., pero no me muero yo, y así se produce la tranquilización respecto a la muerte.

Frente a esto, Heidegger muestra que en el ámbito de la "propiedad (Eigentlichkeit)" la muerte no es un acontecimiento intramundano, sino que es una posibilidad del hombre y por ello se incorpora al ser de éste. La muerte es como posibilidad y, por consiguiente, es justamente cuando nosotros somos y en tanto que nosotros somos. La muerte es ya ahora como posibilidad, como todavía-no (sólo la muerte como acontecimiento óntico se produce cuando nosotros ya no somos).

En resumen, lo que muestra Heidegger es que en las esencias distintas del hombre el todavía-no debe entenderse como falta. Al añadir lo que falta la esencia se completa. Por el contrario, al añadir al hombre todo lo que falta, no se completa la esencia, pues, más bien, lo que sucede es que desaparece el todavía-no y con él desaparece el hombre: esto es la muerte.

\section{Conclusión}

Es claro que a Heidegger le cabe el mérito de sacar a la luz el tema del hacerse propio del ser humano. Pero su teoría en principio muy brillante y sugerente no llega a solucionar el problema del hombre, porque elimina la 
esencia. El error básico de la teoría heideggeriana, que detecta muy bien $\mathrm{Zu}-$ biri, es transformar toda la esencia en existencia. Cualquier intento de prescindir de la esencia ha de conducir al fracaso, porque supone entender al hombre sólo como todavía-no, es decir, como mero no-ser. Frente a este nihilismo heideggeriano, lo que he defendido, utilizando la ontología de Zubiri, es que en el hombre tiene que haber parte de ser, tiene que haber una esencia, que permita incorporar el no-ser también como esencial.

Sólo cuando contamos con la esencia, la brillante descripción existencialista cobra todo su valor. Lo peculiar del hombre es que su esencia no está ya de antemano totalmente configurada. El hombre posee una esencia constitutiva, que es una esencia abierta, abierta al poder ser, al todavía-no. Debido a la apertura, a la esencia inicial constitutiva se añaden otras notas, las constitucionales, que, aun siendo esenciales, son variables (por haber sido incorporadas, por no estar dadas de antemano). Así, la esencia constitutiva, que es la esencia en sentido clásico, incorpora el cambio, dando lugar a una esencia constitucional - esencia en el nuevo sentido aquí propuesto - .

Las descripciones heideggerianas muestran que en el hombre la apertura resulta esencial, porque cuando el todo humano logra incorporar realmente todas sus partes, cuando todo el todavía-no, el futuro, se convierte en un ya-sí, en pasado, entonces la esencia se cierra... y esto no es otra cosa que la muerte. ${ }^{24}$ Pero frente a la primacía del futuro que se postula en el existencialismo, lo que he intentado justificar aquí es que hemos de recuperar el valor irrenunciable de la esencia... y, por tanto, del pasado. ${ }^{25}$ El futuro es importante. Pero para que la persona pueda hacerse en el futuro es necesario que sea algo ya desde siempre: esto constituye lo que podemos denominar el pasado absoluto de la esencia constitutiva, de una esencia que es siempre ya pasado. Además, si la vida humana no ha de ser una multiplicidad de aconteceres inconexos, hay que contar no sólo con el futuro, sino con los futuros ya acontecidos, ya pasados, pues lo que lo que el individuo ha hecho ya de sí en sus futuros anteriores no puede desaparecer, sino que ha de irse añadiendo a la esencia. Así tenemos un pasado incorporado en el presente, que constituye el nivel constitucional de la esencia.

En una palabra, con la nueva ontología que he esbozado aquí, entendemos a la persona como una esencia dinámica. Frente al existencialismo, la persona no es un mero acontecer del ser, no es una pura nada abocada a la muerte, sino una esencia que puede estar dotada, como quería Scheler, de un

24 En el ámbito intramundano, habría que añadir, pues en principio la muerte es un cierre total sólo cuando nos limitamos a dicho ámbito (al que Heidegger circunscribe explícitamente sus análisis). El problema de fondo es si la ontología heideggeriana permite ir más allá de lo intramundano.

25 Y también del presente, pues lo que olvida el existencialismo es que el futuro se abre necesariamente en el ahora, en un presente capaz de incorporar el pasado. 
ordo amoris individual. ${ }^{26}$ Gracias a su peculiar ordo amoris la persona queda abierta a un ámbito individual de valores, que marca metas intransferibles a su hacerse en libertad.

\begin{abstract}
Esta ponencia ofrece el esbozo de una posible "ontología fenomenológica de la persona". El punto de partida es la aceptación del reto existencialista que exige contar con el carácter procesual o dinámico de la vida humana. Pero lo que se defiende, frente al existencialismo heideggeriano, es que la importancia del hacerse, de la existencia no debe llevar a negar la esencia. Para poder hablar de una esencia humana que se hace en parte a sí misma, se utiliza la "teoría de la sustantividad" propuesta por el filósofo español Xavier Zubiri, que, a su vez, se interpreta como una "teoría de todos y partes" del tipo de la desarrollada por el fundador de la fenomenología, Edmund Husserl.
\end{abstract}

${ }^{26}$ La "angustia" ante la muerte deja paso al "amor" que nos abre a un ámbito individual de valores (cf. Scheler, Späte Schriften, Gesammelte Werke, Band 9, zweite, durchgesehene Auflage, Bouvier Verlag, Bonn, 1995, pp. 254-340, donde encontramos la crítica que hizo Scheler a Ser y Tiempo - libro publicado un año antes de su muerte-). Además, como las metas marcadas por valores son supramundanas permiten entender la tesis de que la muerte no constituya la última posibilidad. 